\title{
Direct determination of midplane background neutral density profiles from neutral particle analyzers
}

\author{
K Bogar ${ }^{1,2}$, B Geiger ${ }^{3}$, P A Schneider ${ }^{4}$, A Jansen van Vuuren ${ }^{4}$, \\ O Grover ${ }^{1,5}$, the ASDEX Upgrade team ${ }^{6}$ and the EUROfusion \\ MST1 team $^{7}$ \\ ${ }^{1}$ Institute of Plasma Physics of the CAS, CZ-18200 Praha 8, Czechia \\ ${ }^{2}$ FMP, Charles University, Ke Karlovu 3, CZ-12116 Praha 2, Czechia \\ ${ }^{3}$ University of Wisconsin, Madison, USA \\ ${ }^{4}$ Max Planck Institute for Plasma Physics, Garching D-85748, Germany \\ ${ }^{5}$ FNSPE, Czech Technical University in Prague, CZ-11519 Praha 1, Czechia \\ ${ }^{6}$ See author list of H. Meyer et al. 2019 Nucl. Fusion 59112014 \\ ${ }^{7}$ See author list of B. Labit et al. 2019 Nucl. Fusion 59086020 \\ E-mail: klara.bogar@ipp.cas.cz
}

August 2020

\begin{abstract}
Here we present a new method that allows the determination of background neutral density profiles based on measurements from neutral particle analyzers (NPA). Bayesian optimization is used to obtain a reliable 5-parameter representation of the inferred profiles. The method has been benchmarked using forward modelling from FIDASIM against measured data. The systematic errors coming from assumptions made in the analysis were evaluated and they are lower than $\lesssim 5 \%$.

The new method has been tested using data from the ASDEX Upgrade tokamak. When using reconstructed neutral density profiles, good agreement is found between the measured NPA fluxes of neutralized fast ions with predictions based on TRANSP and FIDASIM. Moreover, a clear drop of neutral density is observed at the plasma boundary after edge localized mode (ELM) activity. As suggested by KN1D simulations, this drop is mainly due to an increase of scrape-off layer (SOL) ionization rate, resulting from higher SOL plasma densities and temperatures after the ELM crash. Moreover, the new method allows calculating the local plasma ionization source which will be important for future transport studies.
\end{abstract}

Keywords: background neutral density, neutral particle analyzer, FIDASIM, ELM

\section{Introduction}

Charge exchange reactions (CX) between fast and thermal plasma ions with background neutrals contribute to the transport across flux surfaces leading to heat and momentum 


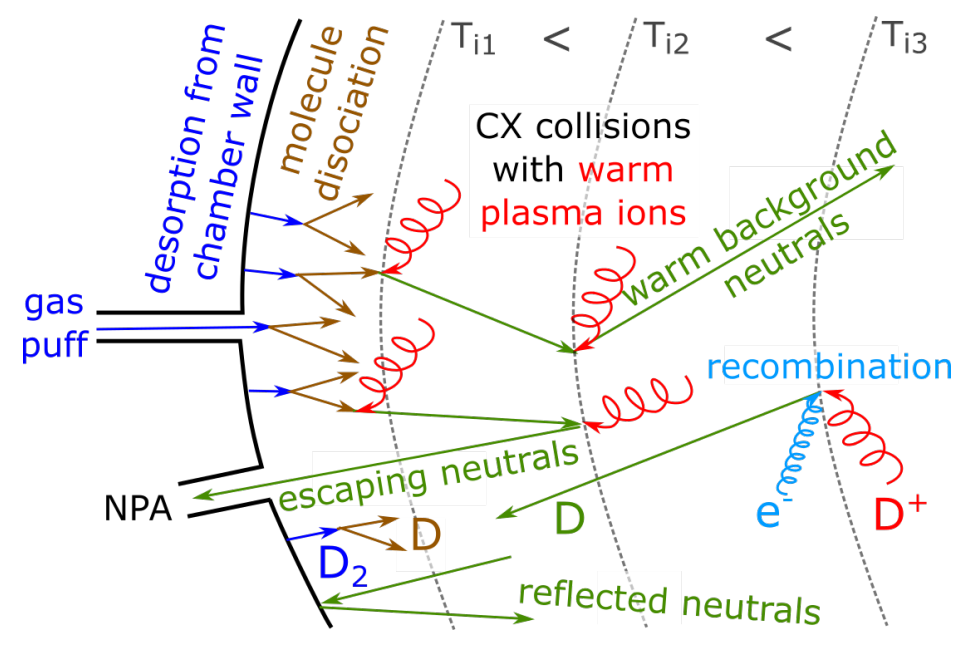

Figure 1. A diagram of different sources of background neutrals inside plasma. Molecular gas comes from the gas puff or wall desorption (blue arrows), cold atoms (brown arrows) with energy $\sim 2 \mathrm{eV}$ are a product of the dissociation process, while multi-step collisions with plasma ions (red) or recombination of plasma ions and electrons (light blue) are sources of warm neutrals (green arrows) capable of penetrating even deep into the plasma. The neutral reflection from the wall can occur for both, cold or warm neutrals. As neutrals are more likely formed in the colder region than at a place, where they undergo the next CX-collision, the local background neutral temperature is lower than the local ion temperature.

losses in fusion devices. Simultaneously, as neutrals interact with a plasma, they become ionized and therefore provide the main particle fuelling source to the plasma. Therefore, accurate knowledge of the background neutral density profiles will improve plasma transport studies, as the plasma ionization source is a missing quantity in the diffusion equation. Also, the analysis of CX losses of fast ions, and the interpretation of measurements of the fast-ion distribution function from fast ion D-alpha spectroscopy or high-energy neutral particle analyzes, require detailed knowledge on the background neutral density as was stressed in [1].

In fusion plasmas, there are several sources of background neutrals which are schematically depicted in figure 1. Plasma fuelling arises from the use of dedicated gas-valves close to the vessel walls and by gas desorption from the chamber wall (or less common pellet injection). The gas molecules, usually $\mathrm{D}_{2}$, dissociate into atoms and ions as they interact with plasma. Formed neutral atoms have low energy $\sim 2 \mathrm{eV}$ [2], therefore they cannot directly penetrate deep into a plasma. However, via multi-step charge exchange collisions with plasma ions, neutrals can propagate into the plasma core. The other sources of neutral atoms are the recombination of plasma ions and electrons and reflection of escaping atoms from the vessel wall. Additional sources of neutrals inside the plasma can be applied, but they are more localized, such as neutral beam injection (NBI), which produces neutrals in the vicinity of the NBI path called halo neutrals, or pellet injection. However, this article is focused on background neutrals, therefore we will not consider NBI halo neutrals or pellets. 
Nowadays, there are several diagnostics providing information about background neutrals, such as pressure gauges [3], Balmer [4] or Lyman alpha spectroscopy [5]. However, these diagnostics are typically not sensitive to background neutrals deeper inside the plasma. Here we present a novel method for determining the whole background neutral density profiles $n_{0}$ directly from neutral fluxes $\Gamma$ measured by the neutral particle analyzers [6].

Neutral particle analyzers (NPAs) measure energy spectra of neutrals escaping the plasma with energies ranging from hundreds of $\mathrm{eV}$ up to hundreds of $\mathrm{keV}$ [6]. In addition, they often have an ability to distinguish between the isotopes such as deuterium and hydrogen. Only neutrals with velocity vectors aiming at the NPA's entrance slit can contribute to the measured flux. In case of so-called passive measurements, when the NPA line of sight does not intersect a neutral beam path, the escaping neutrals are dominantly born by CX-collisions between background neutrals and plasma ions. We are assuming that the reflected neutrals have low probability to aim for the NPA entrance slit and that the neutrals resulting from direct recombination are negligible in comparison with CX neutrals [2].

The measured neutral flux $\Gamma$ can be expressed as a line integral of the emissivity along the NPA line of sight.

$$
\begin{gathered}
\Gamma\left(E_{n}\right)=\int_{0}^{L} \operatorname{emissivity}\left(E_{n}, l\right) \mathrm{d} l \\
=A \int_{0}^{L} n_{0}\langle\sigma v\rangle_{\mathrm{CX}} n_{\mathrm{i}}\left(E_{n}, p, l\right)\left(\int_{0}^{l}\left(\lambda_{\mathrm{att}}\left(E_{n}, x\right)\right)^{-1} \mathrm{~d} x\right) \mathrm{d} l
\end{gathered},
$$

where $E_{n}$ is the measured energy, $A$ is a geometrical factor including NPA aperture and opening angle, $L$ is the length of the NPA line of sight, $n_{0}$ is the background neutral density including all types of the background neutrals presented inside the plasma (products of CX or recombination reaction or reflection from the vessel surface), $\langle\sigma v\rangle_{\mathrm{CX}}$ is the CX collision rate and $n_{\mathrm{i}}\left(E_{n}, p, l\right)$ is the density of ions with velocity vectors directed towards the NPA. Here, $p$ stands for pitch, which is the ratio of the velocity parallel to the magnetic field to the total velocity $v_{\|} / v_{t o t}$. Finally, $\lambda_{\text {att }}\left(E_{n}, x\right)$ represents the mean free path of the formed neutral, as it can undergo re-ionization collision with plasma particles and $l$ is a coordinate along the NPA line of sight.

The parameter $n_{i}\left(E_{n}, p, l\right)$ in equation (1) is established by a local shifted Maxwellian distribution when considering a thermal ion distribution. The latter depends on the local ion temperature $T_{\mathrm{i}}$, plasma rotation $v_{\text {rot }}$, plasma density $n_{\mathrm{e}}$ and plasma effective charge $Z_{\text {eff }}$. Since the charge exchange recombination spectroscopy (CXRS) become standard routinely operated diagnostic at most of the tokamaks which provides detailed information on ion temperature $T_{\mathrm{i}}$ and plasma rotation $v_{\text {rot }}$ profiles including edge plasma region [7], [8], [9], the background neutral density along NPA lines of sight is the only unknown quantity in (1).

First NPAs were mainly used for determination of the ion temperature from the slope of their energy spectra in logarithmic scale. As the isotope separation became 
available, the NPAs started to be exploited also for the isotope ratio determination. The NPAs are also available to measure energy spectra in orders of $100 \mathrm{keV}$ and therefore they are widely used to study fast ions behaviour [10]. However, the background neutral density entering NPA data analysis has been usually derived by simulation codes such as TRANSP, KN1D or DOUBLE [11], [12], [13], [14].

This paper is structured as follows: Section 2 provides a detailed description of the new method determining background neutral densities, including a discussion of each term of equation (1) and the required assumptions. Section 3 then shows an exemplary case, where the outputs and associated systematic uncertainties of the method are examined. The temporal evolution of the midplane background neutral profile and plasma ionization source during an averaged ELM cycle are presented in the section 4. Possibilities how to improve the quality of the method outputs and discussion of the obtained results are finally presented in the section 5 .

\section{Method}

The neutral flux defined by the integral in equation (1) can be expressed by the following matrix representation:

$$
\vec{\Gamma}\left(E_{n}\right)=\hat{M}\left(E_{n}, l_{m}\right) \overrightarrow{n_{0}}\left(l_{m}\right) .
$$

Here the flux $\vec{\Gamma}\left(E_{n}\right)$ has $n$ elements, which correspond to the number of energy bins of a given NPA detector. The matrix $\hat{M}$ contains the geometrical factor $A$, the CX collision rate, the ion density, the attenuation term and $\mathrm{d} l$ as introduced in equation (1). The vector $\overrightarrow{n_{0}}\left(l_{m}\right)$ has $m$ elements, where $m$ describes the individual steps along a given line of sight.

To derive the background neutral density along each step $\overrightarrow{n_{0}}\left(l_{m}\right)$, we need to solve the inverse problem. In the vicinity of the separatrix $(\rho=1)$ steep fall off of the background neutral density is expected. Therefore, the number of $m$ steps should be at least in the order of tens, but optimally few hundreds. Note here that the computational requirements grow up with the numbers $m$ and $n$. To minimize the number of $m$ steps, we have chosen a non-linearly spaced representation with the mapped points being more tightly distributed closer to the NPA, where the steep gradient in the plasma profiles is expected. The number of energy bins $n$ are set by the number of NPA detector channels. The number of useful measured points of $\Gamma_{\text {th }}$ is usually $n \sim 10$ [15], [16], [13]. The mentioned conditions cause that the matrix $\hat{M}$ is not well invertible and it makes our problem ill-posed.

Nowadays, there are several methods to solve ill-posed problems. Here, we employ the Bayes theorem, combined with the Monte-Carlo Markov chain method as implemented in the PyMC3 library [17]. The Bayes theorem (also shown in equation (3)) states that the probability of physics quantity parameters $\left(n_{j}\right)$ properly describing the measured data $\left(N_{k}\right)$, is proportional to the product of the probability that such data are measured in a system, times the probability of occurrence of such parameters. In addition, $I$ refers to additional available information. Usually, the left hand side term in 
equation (3) is known as the posterior, the two terms on the right hand side are called likelihood and prior, respectively.

$$
P\left(\left\{n_{j}\right\} \mid\left\{N_{k}\right\}, I\right) \propto P\left(\left\{N_{k}\right\} \mid\left\{n_{j}\right\}, I\right) \times P\left(\left\{n_{j}\right\} \mid I\right) .
$$

In our case, we describe $n_{0}$ by a parametric function. Therefore, the posterior distribution represents the probability of $n_{0}$ parameters. The likelihood function is described by a Poisson distribution with observations equal to the measured count rates and the prior is a set of uniform distributions of parameters representing $n_{0}$. The Poisson distribution is chosen because it describes count rates as measured by NPA. The prior is chosen such that the background neutral density $n_{0}$ is monotonically increasing with the normalized poloidal flux, $\rho$.

To describe background neutral density $n_{0}$, a modified hyperbolic tangent function is defined using five parameters: $r_{2}, e_{2}, e_{1}, n_{0, \text { ax }}$ and $n_{\text {sep }}$.

$$
\begin{gathered}
f(\rho)=\left(\rho^{1.5}-r_{2}^{1.5}\right) e_{2} \quad g(\rho)=\frac{e_{1}(1-\rho)+\exp \left(e_{1} \rho\right)}{1+e_{1}} \\
\log _{10}\left(n_{0}\right)=n_{0, \text { ax }} \frac{g(\rho) \exp (-f(\rho))+n_{\text {sep }} \exp (f(\rho))}{\exp (f(\rho))+\exp (-f(\rho))},
\end{gathered}
$$

where $r_{2}$ and $e_{2}$ define the drop of $n_{0}$ close to the separatrix. The inner part of the profile is described by an exponential decay $g\left(\rho, e_{1}\right)$ towards the central value $10^{n_{0} \text {, ax }}$, where we impose that $\delta n_{0}(\rho=0) / \delta \rho=0$. The edge neutral density corresponds to $10^{n_{0, \text { ax }}+n_{\text {sep }}}$. To obtain background neutral density $n_{0}$ along the NPA line of sight, $\overrightarrow{n_{0}}\left(l_{m}\right)$, we assume that the neutral density is constant on flux surfaces. This is not generally valid as the density of background neutrals can vary poloidally by orders of magnitude [18]. The reconstructed background neutral density profiles are therefore only valid in the vicinity of the NPA line of sight.

Inputs for the Bayes inversion are the measured count rates of $\Gamma_{\text {th }}$ and the matrix $\hat{M}\left(E_{n}, l_{m}\right)$. The individual terms needed to prepare $\hat{M}$ are obtained based on the following assumptions:

- Tabulated CX collisional rates $\langle\sigma v\rangle_{\mathrm{CX}}$ as used in FIDASIM [11] are applied which include information on the population of excited states of newly formed neutrals. Here, we assume that the background neutrals are all in the ground state and that their temperature is equal to the local ion temperature. The impact of this assumption is discussed in section 3.

- The local ion distribution function $n_{\mathrm{i}}\left(E_{n}, p, l\right)$ with energy $E_{n}$ and pitch $p$ oriented towards the NPA is defined by the Maxwellian distribution of plasma ions with temperature $T_{\mathrm{i}}$ shifted by the plasma rotation velocity $v_{\text {rot }}$. We assume that the dominant term of the plasma rotation is the toroidal rotation, where toroidal stands for the parallel direction to the magnetic field vector on the magnetic axis. The total ion density profile is based on measured plasma electron density $n_{\mathrm{e}}$ and effective charge $Z_{\text {eff }}$. 


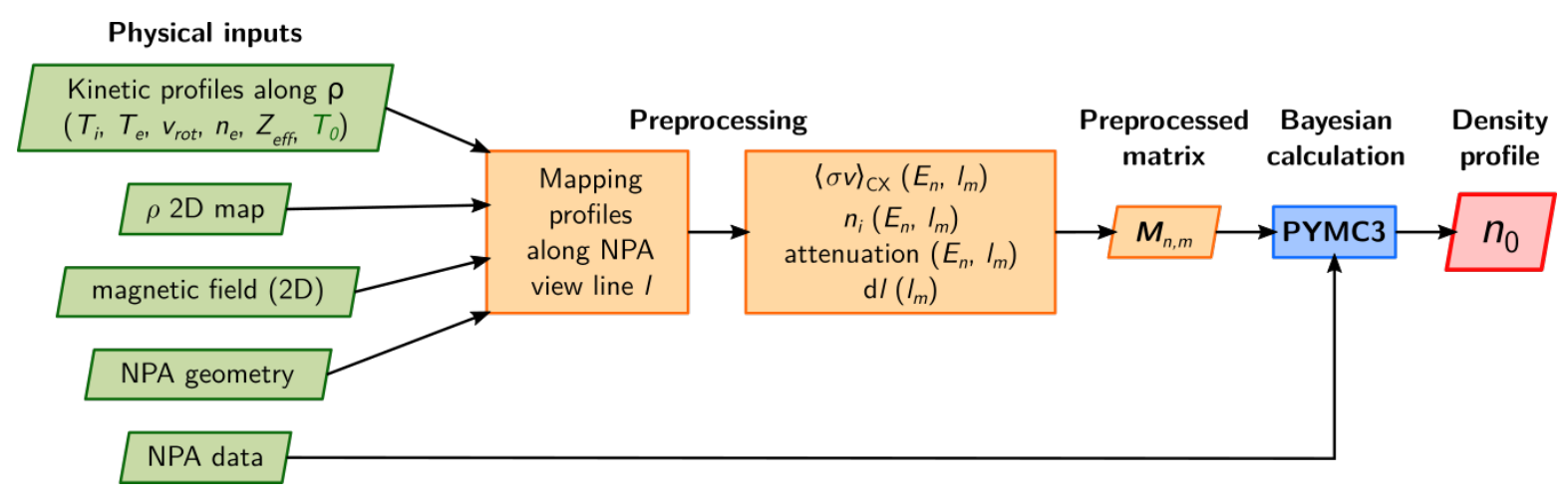

Figure 2. A diagram of $n_{0}$ fitting routine. 1D kinetic profiles as a function of $\rho$ are mapped along a given NPA line of sight $l$ using a 2D map of rho-values. 2D magnetic field map is used to determine the relevant pitch values along the NPA line of sight $l$. When all profiles are mapped, the individual terms of equation (1) are derived for each energy bin and step along $l$, employing FIDASIM cross sections. These terms define elements of the matrix $\hat{M}_{\mathrm{n}, \mathrm{m}}$. Finally, the measured NPA count rates and $\hat{M}_{\mathrm{n}, \mathrm{m}}$ are input into the Bayesian calculation based on PYMC3 to obtain $n_{0}$ density profiles.

We do not consider a deviation of the actually measured impurity temperatures and rotations by CXRS from the bulk plasma ion temperatures and rotations. We also neglect possible anisotropy of the ion temperature. Both of these effects were presented in [19]. However once more detailed information about the bulk plasma ion temperatures and rotations will be routinely available, the input bulk plasma ion distribution into our method can be easily modified.

- To get the attenuation term $\int_{0}^{l}\left(\lambda_{\text {att }}\left(E_{n}, l\right)\right)^{-1} \mathrm{~d} x$, the collisional radiative model of FIDASIM is used. This model accounts for excited atomic states up to $\mathrm{n}=6$. Population of excited states after CX collisions is taken into account.

- Relative collisional energy shift as a consequence of plasma rotation is considered for all collisions.

- The NPA line of sight is considered as a single line, which is in reality a narrow cone defined by the NPA slits geometry. The real geometry is considered by FIDASIM, which is used for the benchmark of our results.

Figure 2 summarizes the individual steps needed to obtain $n_{0}$ profiles using a flow chart.

\section{Example case}

The new method of determining background neutral densities has been tested using experimental data obtained at ASDEX Upgrade during discharge \#32065 at $~ 5.5 \mathrm{~s}$. This experiment is a deuterium $\mathrm{H}$-mode single null diverted discharge. The plasma parameters for the given time are the following: $B_{\mathrm{t}}=-2.47 \mathrm{~T}, I_{\mathrm{P}}=0.65 \mathrm{MA}$, $n_{\mathrm{e}, \text { core }}=4 \times 10^{19} \mathrm{~m}^{-3}, Z_{\mathrm{eff}}=1.6, P_{\mathrm{NBI}}=2.4 \mathrm{MW}$. The selected time is already in the ramp-down phase of the discharge, but this should not have any impact on the 


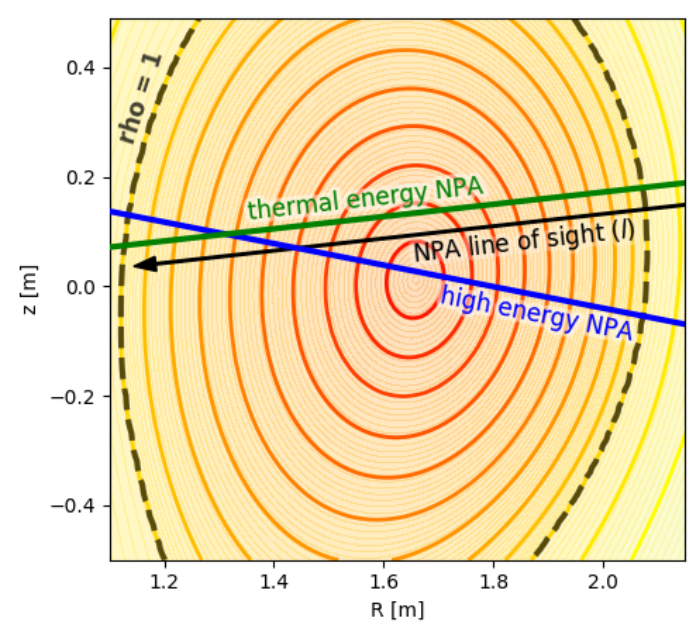

Figure 3. Lines of sight geometry of NPAs at ASDEX Upgrade. The upper NPA collects thermal neutrals and is used to reconstruct background neutral density profile, while the lower detector was set to high energies. The coordinate along the thermal NPA line of sight starts at the detector position and its direction is highlighted by a black arrow. In addition, the flux-surfaces of discharge \#32065 are shown.

quality of presented results. A poloidal view on the flux surfaces and the NPA's lines of sight are depicted in figure 3, while measured kinetic profiles as a function of the distance along the NPA's line of sight are show in figure 4.

As can be seen, ASDEX Upgrade is equipped with two NPAs with variable energy range settings [15]. For the study presented here, one NPA was set to measure the thermal neutral flux $\Gamma_{\text {th }}$ (low energies) while the second NPA was set to high energies to observe neutrals originating in CX collisions of background neutrals and non-Maxwellian fast ions $\Gamma_{\mathrm{fi}}$, a consequence of NBI heating.

The background neutral density profile deduced by our novel method $n_{0_{\mathrm{NPA}}}$ is presented in figure 5 a). To validate the result shown in figure 5 a), the obtained background neutral density profile $n_{0_{\mathrm{NPA}}}$ has been used as an input to FIDASIM [11], which is a Monte Carlo code on a 3D simulation grid that allows the calculation of synthetic NPA signals. The excellent agreement between the resulting synthetic thermal NPA fluxes from FIDASIM and the measurements shown in figure $5 \mathrm{~b}$ ) validates the new method (red line vs. green points). The remaining slight disagreement is likely explained by the fact that we did not take into account the differences in the numerical approaches between our method and FIDASIM. For example, the size of the grid step $\mathrm{d} l$ differs, it is uniform in case of FIDASIM and increasing in our case as seen in figure $4 \mathrm{c}$ ). The additional source of potential deviations in the case of high energy NPA in figure $5 \mathrm{c}$ ) is the fast-ion distribution provided by TRANSP. The fast-ion distribution was obtained with the assumption of the neoclassical transport of fast-ions, which was shown in [21] to be close to the observations. To prove a sufficient sensitivity of FIDASIM to the input background neutral density profile, there are FIDASIM outputs presented in figure $5 \mathrm{~b}$ ) for two modified background neutral densities, one with third core value (black dashed 

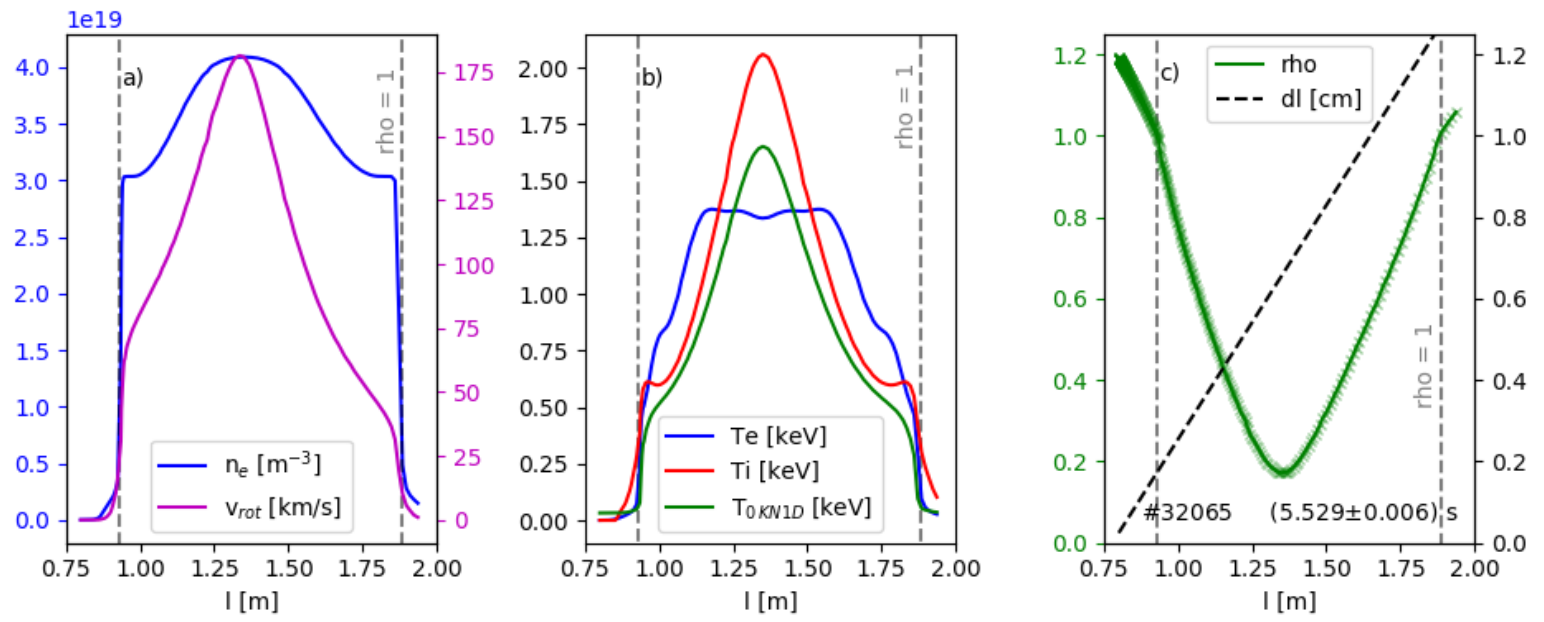

Figure 4. Kinetic profiles of \#32065 averaged over $12 \mathrm{~ms}$ around $\mathrm{t}=5.529 \mathrm{~s}$ are mapped on the line of sight of the upper (thermal) NPA starting at the NPA detector position: a) Electron density $n_{\mathrm{e}}$ and toroidal rotation $v_{\text {rot }}$, b), electron and ion temperature $T_{\mathrm{e}}$ and $T_{\mathrm{i}}$, c) $\rho$ and differential of the NPA line of sight coordinate $\mathrm{d} l$. In addition, the background neutral temperature $T_{0, \mathrm{KN} 1 \mathrm{D}}$ is shown in b) which is an output of KN1D [20]. The separatrix positions are indicated by the grey dashed lines.
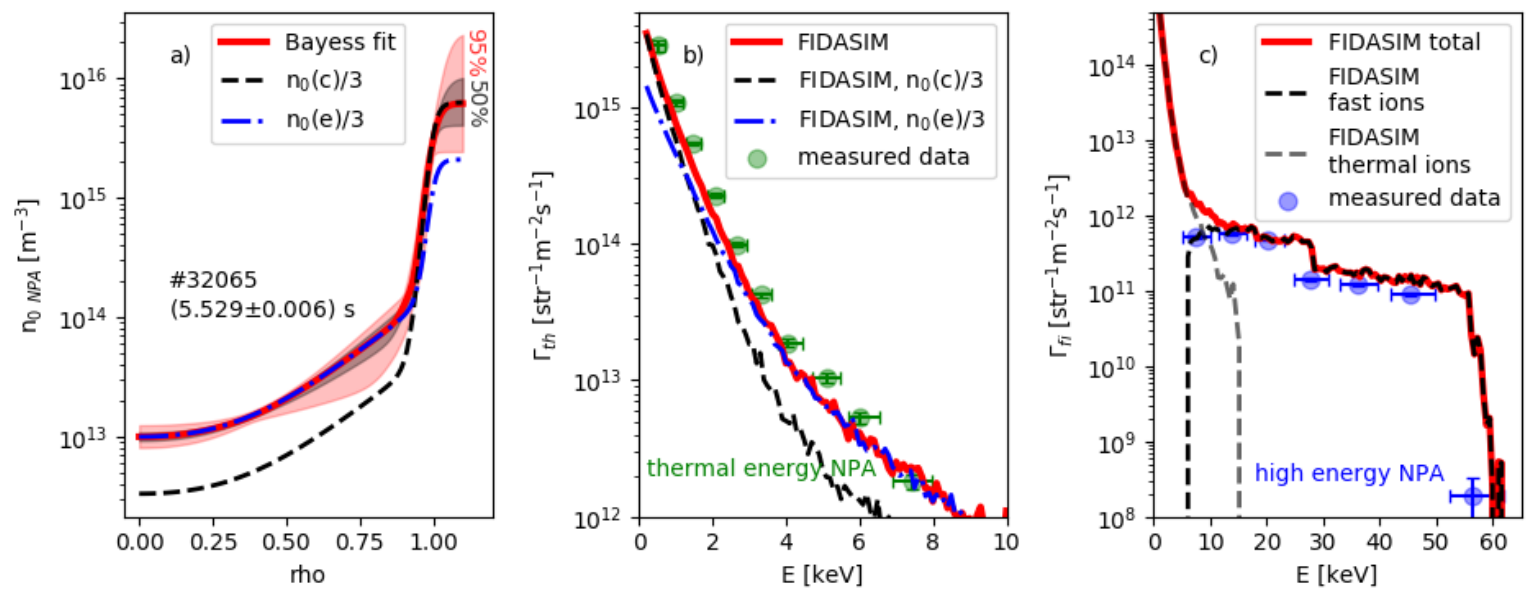

Figure 5. Reconstructed background neutral density profile $n_{0_{\mathrm{NPA}}}$ including areas of $50 \%$ and $95 \%$ certainty is shown in a). Thermal flux, which serves for fitting and its FIDASIM reconstruction are in b). High energy NPA data and its FIDASIM reconstruction are in c). Note that the profile from a) serves as input to the FIDASIM simulations presented in b) and c). To see the sensitivity of FIDASIM on the input background neutral density $n_{0}$ there are two additional FIDASIM outputs for the thermal energy NPA in b) for the background neutral density profile with third core (black dashed) and edge (blue dash-dotted) values, which are depicted in a).

lines) and second with third edge value (blue dash-dotted lines), which are shown in in figure 5 a).

Figure 5 c) shows a comparison between FIDASIM and the NPA measurement of high energetic neutrals $\Gamma_{\mathrm{fi}}$. Here again, reasonably good agreement is found between 


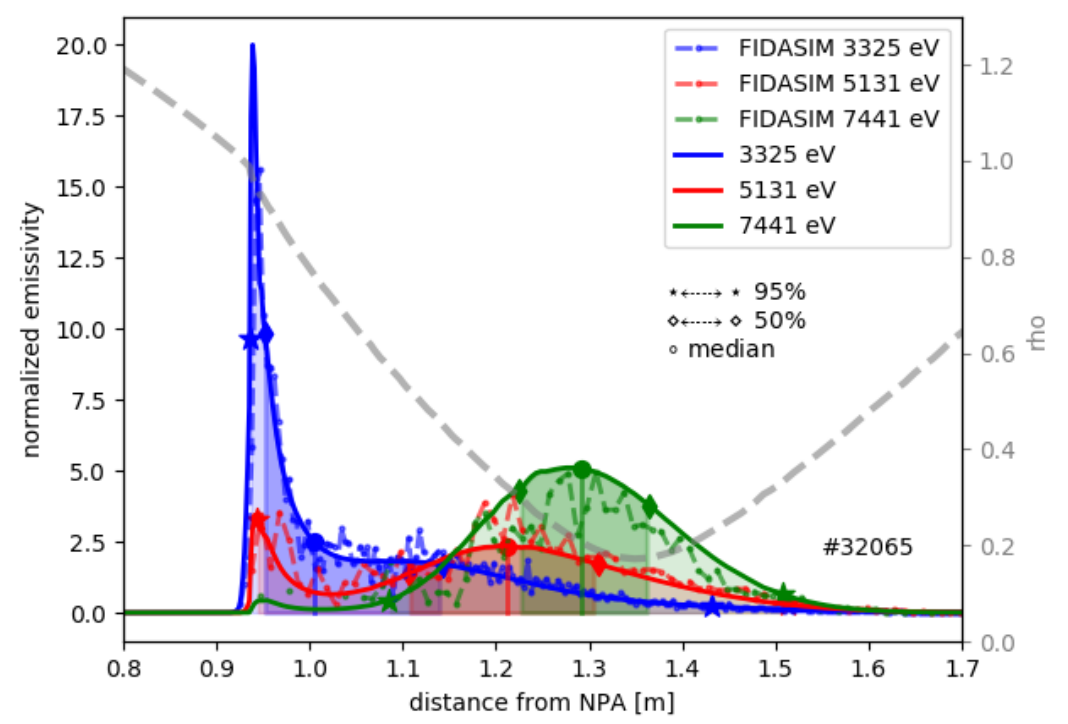

Figure 6. Normalized emissivity, which is defined in (1), along the NPA line of sight. As energy increases, the flux of escaping neutrals originates from deeper inside the plasma. Note here, that the solid lines correspond to the new method, while the dashed lines are predicted by FIDASIM. The grey dashed line is mapped $\rho$ along the NPA line of sight. Dark and light color bands with diamonds and stars on their edges represent regions where $50 \%$ and $95 \%$ of the measured signal comes from. The circles stand for the emissivity median position.

the experimental NPA data and the FIDASIM using the background neutral density $n_{0_{\mathrm{NPA}}}$ profile from the new method. The remaining differences might be explained by uncertainties in the TRANSP predicted neoclassical fast ion distribution function, which has been considered in FIDASIM.

Figure 6 shows the corresponding normalized distance-resolved thermal flux of escaping neutrals, called emissivity as introduced in (1), for different energy ranges. Emissivity is determined with the obtained background neutral density profile in figure 5 and counted terms of matrix $\hat{M}$, which are divided by $\mathrm{d} l$. Clearly, high energetic neutrals dominantly originate from the plasma core (large distances to the NPA) while low energy neutrals originate primarily from the plasma periphery. This shows that energy resolved NPA measurements, combined with ion temperature measurements, provide radial information which is exploited by the new method proposed here.

The relatively low uncertainty of the reconstructed background neutral density in figure 5a in the vicinity of $\rho \sim 1$ is explained by significant fluxes of low energy neutrals. Similarly, the region $\rho \sim 0.4$ has also low uncertainty, but due to high energy fluxes coming from this region. In contrast, the high uncertainty around $\rho \sim 0.75$ is a consequence of the local minimum of all emissivities in this region, caused by a significant attenuation of lower energetic fluxes and simultaneously local $T_{\mathrm{i}}$ values that are not high enough to produce more energetic neutrals measured by higher channels. Little 


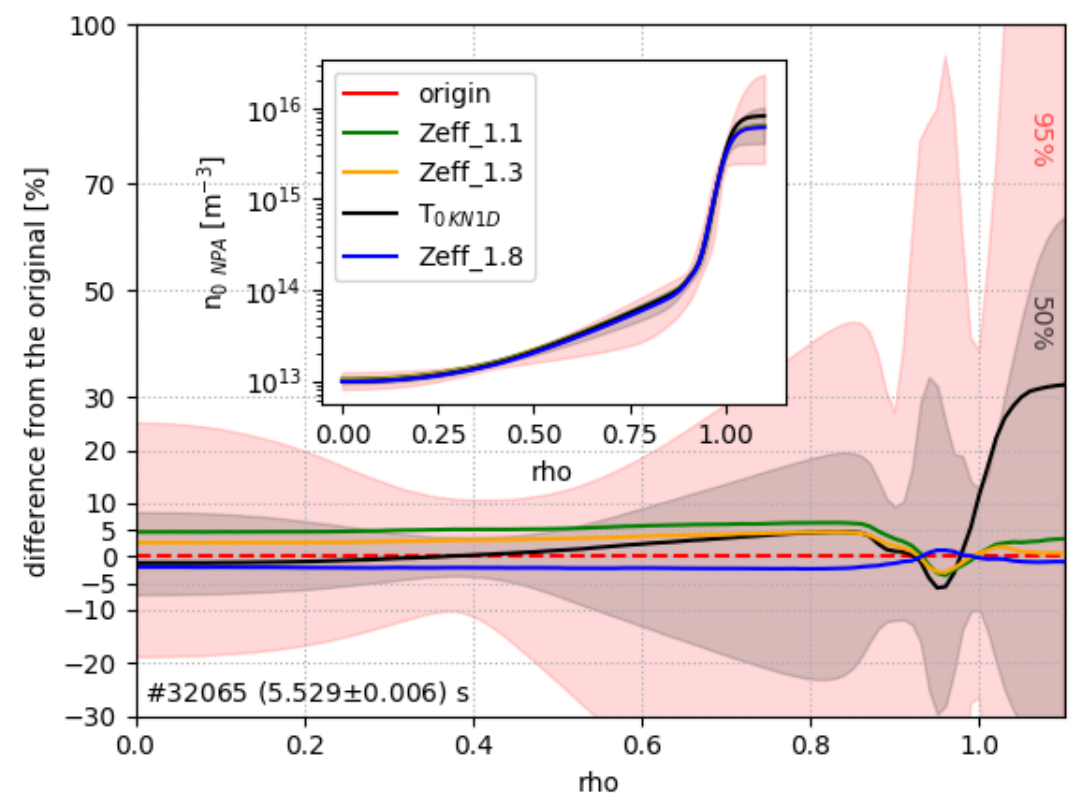

Figure 7. Reconstructed background neutral $n_{0_{\mathrm{NPA}}}$ density for different $Z_{\text {eff }}$ with $T_{\mathrm{i}}$ $=T_{0}$ and neutral temperature $\mathrm{T}_{0 K N 1 D}$ from KN1D, as shown in figure $4 \mathrm{~b}$ ), with $Z_{\text {eff }}$ $=1.6$ and their relative deviation from the origin fit having $Z_{\text {eff }}=1.6$ and assumption $T_{\mathrm{i}}=T_{0}$.

information is available in the SOL $(\rho>1)$ where the plasma is cold and neutral fluxes escaping this region are under the measured NPA energy range. Also the NPA LOS does not cross the plasma centre in this discharge. The quality of the fit relies strongly on input plasma parameters, mainly $T_{\mathrm{i}}$, which governs the population of energetic ions undergoing CX collisions with background neutrals, and $n_{\mathrm{e}}$, which affects escaping neutral flux attenuation.

In the procedure of the background neutral density $n_{0_{\mathrm{NPA}}}$ fitting we assumed that the temperature of the background neutrals $T_{0}$ is equal to ion temperature $T_{\mathrm{i}}$. In reality, however, the background neutrals are a bit colder than ions (see figure $4 \mathrm{~b}$ )). This is a result of the radial transport of the background neutrals from colder regions deeper into the plasma, as depicted in figure 1. To derive the consequent systematic error of the assumption, we employed the 1D neutral density code called KN1D [20], which provides the $T_{0}$ profile. There is also a lack of $Z_{\text {eff }}$ profile measurements, so we simplify it by a constant value $Z_{\text {eff }}=1.6$ over $\rho$. The results when applying the Bayesian fitting method assuming $T_{0}$ from KN1D and for $Z_{\mathrm{eff}}=1.1,1.3$ and 1.8 are shown in figure 7 .

As shown in the figure 7 , the deviation from the original fit caused by the varying $Z_{\text {eff }}$ is below $\sim 5 \%$ everywhere. Moreover, the assumption of $T_{0}=T_{\mathrm{i}}$ is reasonable inside the confined region. In case there will be $T_{0}$ measurements available in the scrape-off layer (SOL) the method could be applied even for this region. It should be noted that uncertainties in the NPA absolute flux calibration directly transfer to the uncertainties of the reconstructed background neutral density $n_{0_{\mathrm{NPA}}}$ absolute values, but they do not 
affect its shape.

\section{Background neutral density profile evolution over ELM cycle}

Having a tool to extract background neutral profiles $n_{0}$, we can reconstruct their temporal evolution over an edge localized mode (ELM) cycle [22]. The ASDEX Upgrade discharge \#36309 has been selected, which features a sufficiently high plasma density such that accurate background neutral density reconstructions are possible in the pedestal region. A list of used diagnostics, observed regions, temporal resolution and references is given table 1 . Representative plasma parameters are shown in figure 8. To receive an accurate behaviour of kinetic profiles in respect to the ELM crash, we have sorted time axes of measured data with respect to the closest ELM crash time as defined by the divertor current signal, which is usually used at ASDEX Upgrade as ELM onset diagnostic. Then we selected only the ELMs, which are highlighted in figure 8 and applied 2D splines ( $\rho$ and time) on the measured kinetic profiles. The obtained behaviour of the ELM synchronised kinetic profiles between $\rho=0.83$ and $\rho=1.1$ are plotted in figure 9 .

Table 1. List of used diagnostics, respective observed plasma regions and their time resolution.

\begin{tabular}{lllll}
\hline quantity & diagnostic & measured location & time resolution & ref. \\
\hline$n_{\mathrm{e}}$ & IDA & all profile & $1 \mathrm{~ms}$ & {$[23]$} \\
$T_{\mathrm{e}}$ & ECE & core & $0.064 \mathrm{~ms}$ & {$[24]$} \\
& Thomson scattering & all profile & $\sim 10 \mathrm{~ns}$ & {$[25]$} \\
\multirow{2}{*}{$T_{\mathrm{i}}$ and $v_{\text {rot }}$} & CXRS CMZ & edge & $2.3 \mathrm{~ms}$ & {$[8]$} \\
& CXRS CEZ & core & $5 \mathrm{~ms}$ & {$[7]$} \\
& CXRS CUZ & core & $10 \mathrm{~ms}$ & {$[7]$} \\
$\Gamma_{\text {th }}$ & NPA & & $1 \mathrm{~ms}$ & {$[6]$} \\
\hline
\end{tabular}

As the gas puff was constant over the selected discharge period, the only varying source of background neutrals is the wall desorption. ASDEX Upgrade is equipped by a set of edge pressure gauges measuring local pressure $p_{0}$ inside the tokamak chamber [26]. The evolution of the midplane neutral pressure $p_{0}$ over the ELM cycle at ASDEX Upgrade was already discussed in [27]. It was shown that the midplane particle flux from the walls is directly proportional to neutral pressure $p_{0}$, increases immediately after ELM crashes as a consequence of the ELM-induced power and plasma particle flux towards the vessel walls. After the ELM, the midplane neutral pressure $p_{0}$ drops even below the preELM value and finally, it slowly recovers to the initial value. Unfortunately, midplane gauge measurements are not available for \#36309, but the measured thermal neutral fluxes $\Gamma_{\text {th }}$ by NPA exhibit the same trends as midplane pressure $p_{0}$. The similarity is probably given by the fact that both diagnostics measure fluxes of neutrals. The 

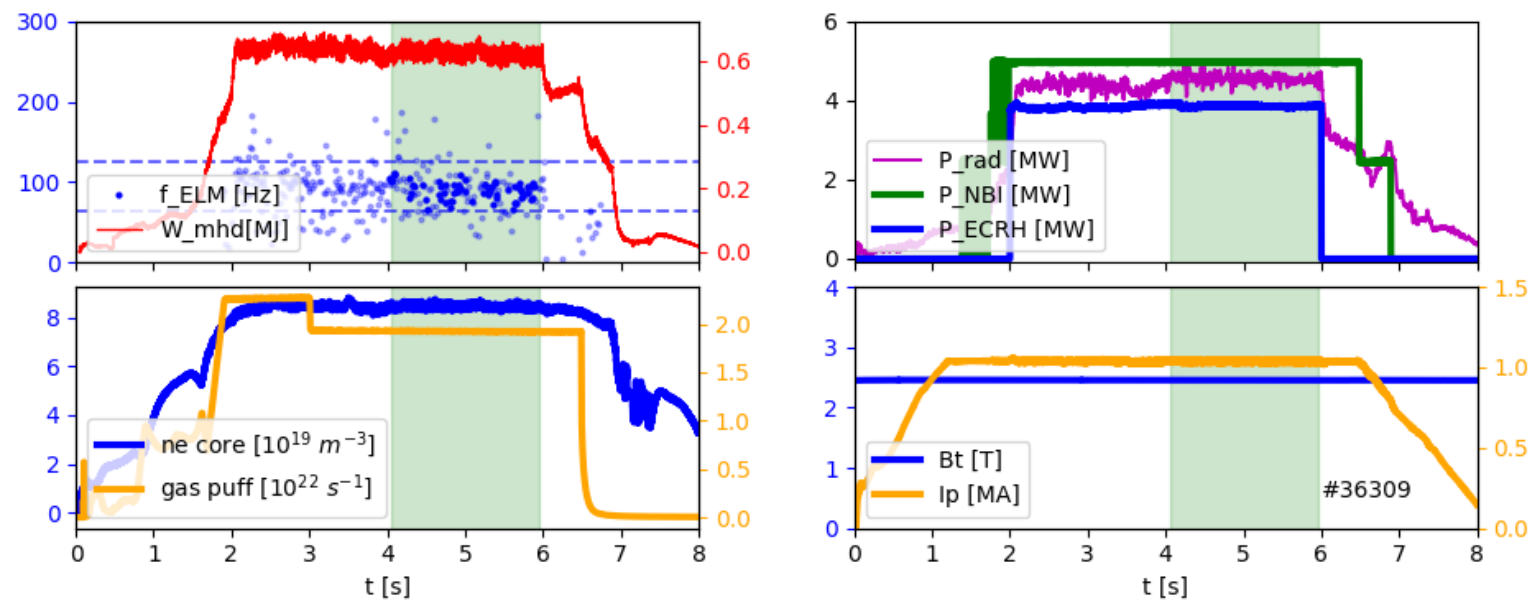

Figure 8. Plasma parameters of \#36309. The highlighted time window from 4.05 to $5.95 \mathrm{~s}$ with stationary conditions was used to average profiles and NPA data in respect to ELM crashes. Only ELMs represented by dark blue points, in between blue dashed lines, in $f_{\mathrm{ELM}}$ were considered. The light blue points were excluded.
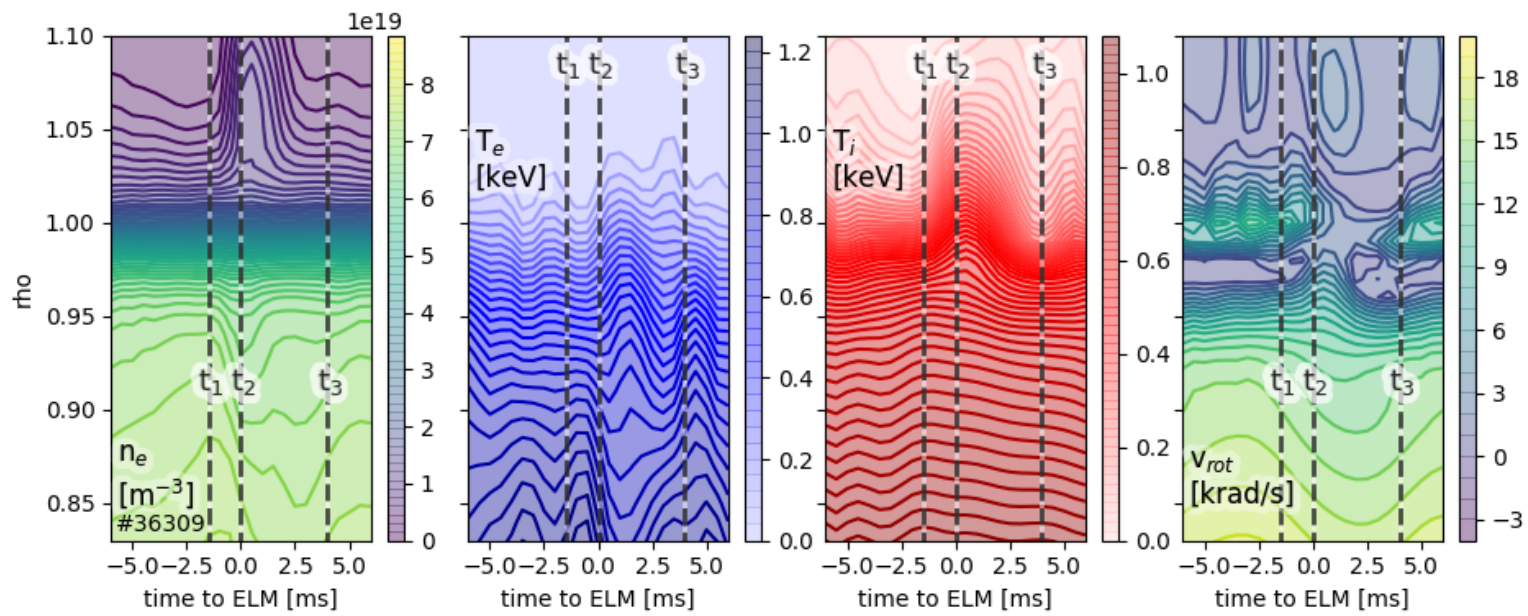

Figure 9. Kinetic profiles behaviour with respect to the ELM crash. The presented profiles are 2D splines of ELM synchronised kinetic profiles of highlighted ELMs in figure 8. As a given ELM crash occurs $\left(t_{1} \rightarrow t_{2}\right)$, the plasma is flushed out of the confined region and the steep gradient (the pedestal) is dramatically reduced. Therefore density and temperatures increase in SOL and simultaneously drop inside the confined region. In case of the electron temperature $T_{\mathrm{e}}$, the border between core drop and edge increase is deeper inside the plasma, $\rho \sim 0.9$ and it is visible $\sim t_{2}$. The ELM crash also flattens the edge plasma rotation. Later, the plasma profiles slowly recover before the next ELM occurs $\left(t_{2} \rightarrow t_{3} \rightarrow t_{1}\right)$. 

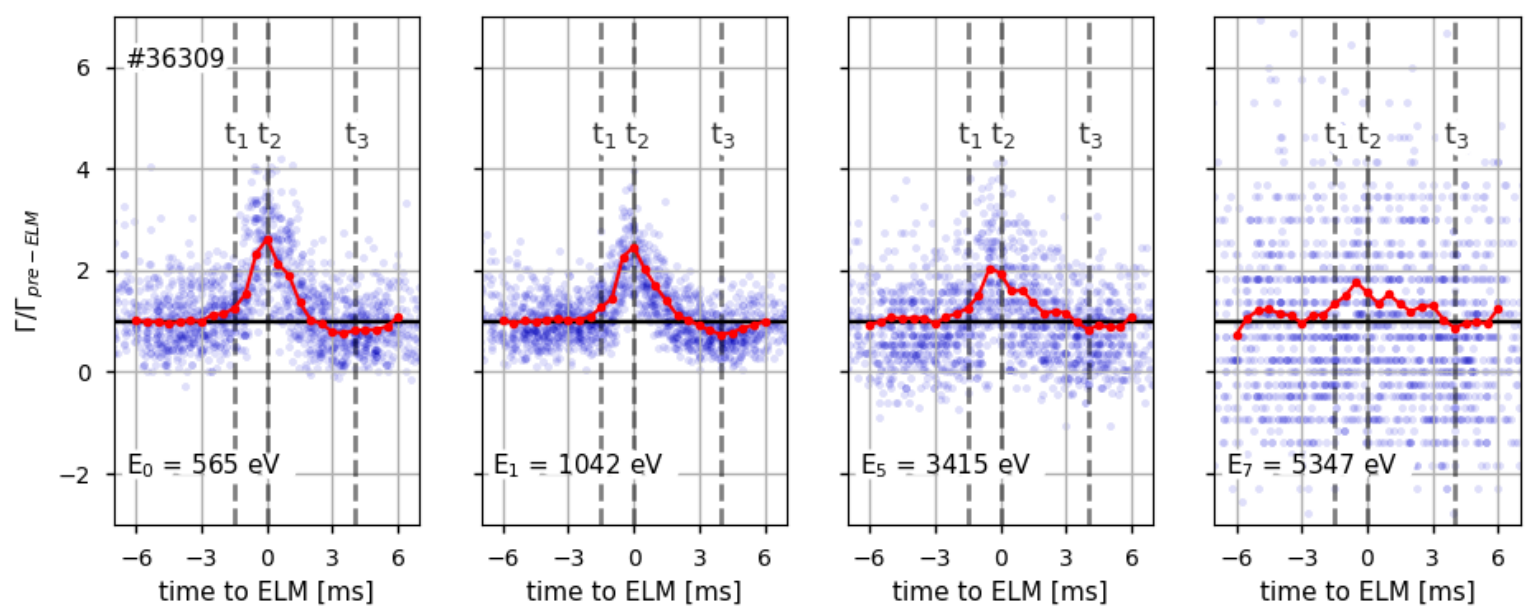

Figure 10. Relative deviation of measured ELM synchronised thermal fluxes from their pre-ELM value for several energy channels are represented by blue dots. The red lines are based on the reconstructed $n_{0_{\mathrm{NPA}}}$.

difference between NPA and pressure gauge is that the gauge does not distinguish energy and kind of incoming neutrals, while the NPA does not detect wall molecules $\mathrm{D}_{2}$ at all.

The temporal evolution of the ELM synchronised thermal neutral fluxes $\Gamma_{\text {th }}$ for several different energies is shown in figure 10 including reconstructed fluxes, based on the reconstructed $n_{0_{\mathrm{NPA}}}$.

There is a significant increase of thermal neutral fluxes $\Gamma_{\text {th }}$ from pre-ELM level accompanying the ELM crash and it is followed by a drop even below pre-ELM value. The ELM signature is more pronounced for low energies than for high energies. Note here that negative fluxes can occur when the signal level is close to the noise level, because the measured background counts are subtracted from raw $\Gamma_{\text {th }}$ counts before $\Gamma_{\text {th }}$ itself is derived. Fortunately, the Bayesian approach allows us to fit the raw $\Gamma_{\text {th }}$ counts directly and to subtract the measured background noise counts as another Poisson distribution.

Based on the thermal neutral fluxes $\Gamma_{\text {th }}$ in figure 10 and the kinetic profiles in figure 9, three essential time points were identified.

- Time $\mathbf{t}_{\mathbf{1}}$ represents the ELM onset, where plasma particles are lost from the confined region and thermal neutral flux $\Gamma_{\text {th }}$ increases rapidly.

- Time $\mathbf{t}_{2}$ corresponds to the maximum of the thermal neutral fluxes $\Gamma_{\text {th }}$ and overlaps with the increase of the divertor current signal, which has been used as the ELM crash reference.

- Time $\mathbf{t}_{\mathbf{3}}$ indicates the minimum of the thermal neutral fluxes $\Gamma_{\mathrm{th}}$.

It is important to note here, that there is a smearing effect from the exposure time of the diagnostics, see table 1 . The least affected is $T_{e}$, based on instantaneous measurements, and the most affected are $T_{\mathrm{i}}$ and $v_{\text {rot }}$, based on the CXRS measurements collecting signals in the comparable time scale as the studied phenomena. 

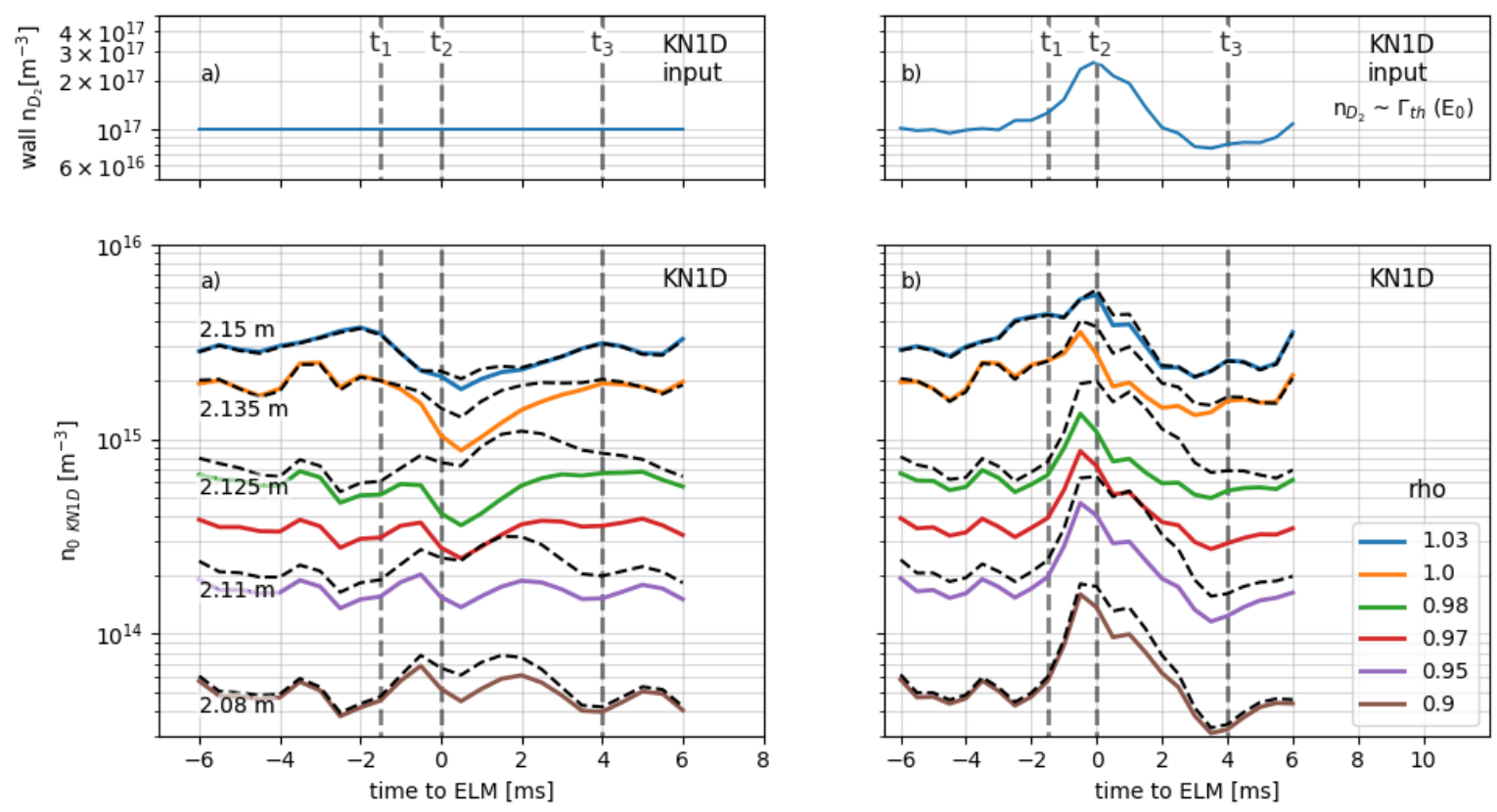

Figure 11. Wall gas densities $n_{\mathrm{D}_{2}}$ (up) serving as KN1D input and corresponding KN1D background neutral densities $n_{0_{\mathrm{KN} 1 \mathrm{D}}}$ (bottom) for different $\rho$ positions (solid colour lines) and fixed $R$ positions (dashed black lines). Case a): There is no impact of the ELM on wall gas density considered. Case b): The wall gas density is expected to be proportional to the lowest measured flux by NPA $\Gamma_{\mathrm{th}}\left(E_{0}\right)$.

The kinetic profiles drive the ionization rate, which is a sink of background neutrals. A source of neutrals is molecular deuterium gas $n_{\mathrm{D}_{2}}$ located at the tokamak wall as well as reflected neutrals figure 1 . The resulting background neutral density profile $n_{0}$ is a result of a balance of source and sink. To study changes of the background neutral density $n_{0}$ during the ELM cycle, which are solely driven by the plasma variation itself, we ran KN1D for each time step with a constant defined wall gas density $n_{\mathrm{D}_{2}}$. Having outputs of KN1D $n_{0_{\mathrm{KN} 1 \mathrm{D}}}$ we can then modify the wall gas density $n_{\mathrm{D}_{2}}$ evolution. As we are missing direct information about the wall recycling due to the absence of a midplane gauge or midplane $\mathrm{D}_{\alpha}$ measurements, we use the NPA's lowest energy channel as mentioned before. The evolution of the background neutral density from $\mathrm{KN} 1 \mathrm{D} n_{0_{\mathrm{KN} 1 \mathrm{D}}}$ during the ELM cycle is shown in figure 11. Moreover, the experimental (reconstructed) background neutral density based on the NPA thermal neutral flux $\Gamma_{\text {th }}$ is shown in figure 12. To show the impact of flux-surface movements during the ELM cycle, we added black dashed lines to the experimental and simulation results which correspond to constant major radii $R$.

The KN1D outputs show that in case of an unaffected neutral source, we would expect a steepening of the background neutral density in the SOL during the pedestal build up before $t_{1}$. The ELM crash would be accompanied by a drop in the pedestal region (see figure $11 \mathrm{a}$ )) between times $t_{1}$ and $t_{2}$ and consequent recovery into pre-ELM values. The deviation of time traces for $\rho=$ const. (color lines) and $R=$ const. (black 

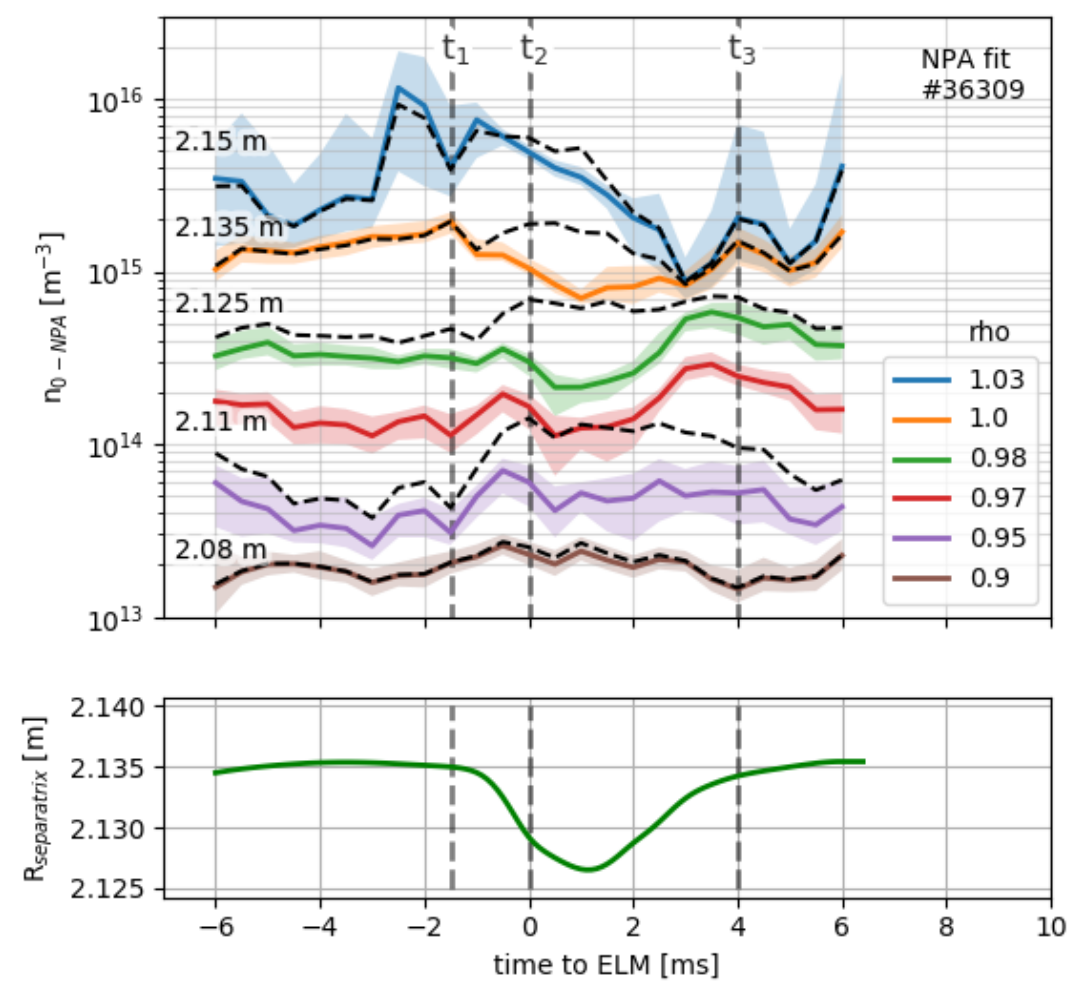

Figure 12. Reconstructed $n_{0_{\mathrm{NPA}}}$ evolution over ELM cycle at different $\rho$ positions (solid color lines) including the $95 \%$ region of certainty (shaded areas) and at different major radius $\mathrm{R}$ (black dashed lines) (up) and separatrix major radius position (bottom).

dashed lines) positions during the ELM crash is a consequence of the radial movement of the plasma boundary and it can account for parts of the drop observed in figure 12 . This effect is significant for region from $\sim \rho=0.97$ to 1 , where the background neutral density drops with the constant $\rho$ position with the ELM crash, while it does not vary dramatically in respect to the constant major radius $R$.

Assuming that wall recycling follows the NPA's lowest energy channel as shown in figure $11 \mathrm{~b}$ ), the evolution of the background neutral density is modified significantly. The increase prior to the ELM in the SOL remains (up to $t_{1}$ ), but it continues up to the ELM maximum, $t_{2}$. The increase between $t_{1}$ and $t_{2}$ appears even inside the confined region. The ELM-accompanying drop starts later $\left(t_{2}\right)$ and continues even below preELM values.

The reconstructed background neutral density evolution based on measured NPA data $n_{0_{\mathrm{NPA}}}$ figure 12 is also increasing before the ELM onset (up to $t_{1}$ ) and drops afterward below the pre-ELM value in the outer region $\left(t_{2} \rightarrow t_{3}\right)$. The reconstructed background neutral density $n_{0_{\mathrm{NPA}}}$ between ELM onset, $t_{1}$, and ELM crash maximum, $t_{2}$, does not drop as dramatically as in the KN1D case with the assumption of a constant wall gas density $n_{\mathrm{D}_{2}}=$ const. (see figure 11 a)) but does not grow as much as in the case with the modified wall gas density. This means, that based on our fitting, the 
wall molecular density grows, but with lower magnitude than the observed thermal flux $\Gamma_{\mathrm{th}}\left(E_{0}\right)$. This is even more visible for the positions deeper inside the plasma, where the impact of the ELM vanishes.

The deviation between the expected wall gas density $n_{\mathrm{D}_{2}}$ based on fitting and thermal neutral flux $\Gamma_{\mathrm{th}}\left(E_{0}\right)$ trends is driven by the fact that thermal neutral flux $\Gamma_{\mathrm{th}}\left(E_{0}\right)$ does not rely only on neutrals, but it is also sensitive to the ion temperature $T_{\mathrm{i}}$ changes. Therefore, we also measure an increase of the background neutral density $n_{0_{\mathrm{NPA}}}$ in the pre-ELM phase, even though the thermal neutral flux $\Gamma_{\mathrm{th}}\left(E_{0}\right)$ is almost constant. In addition, the uncertainty due to the assumption of same neutral and ion temperatures $T_{0}$ $=T_{\mathrm{i}}$ has to be considered. As shown in section 3, figure 7, the reconstructed background neutral density in the SOL is underestimated, because reconstructed background neutral density is higher in the SOL if we use more accurate $T_{0}=T_{0 \mathrm{KN} 1 \mathrm{D}}$ instead of $T_{0}=T_{\mathrm{i}}$, but does not overcome a region of fit certainty.

This study shows that the behaviour of the neutral density depends on the competition between the ionization rate and the wall recycling, i.e. the influx of $\mathrm{D}_{2}$ molecules. The KN1D studies in figure 11 show, for instance, that if the ELM impact on the influx of $\mathrm{D}_{2}$ molecules would be stronger, the ELM crash could be accompanied by an increase of the neutral density rather than slight drop as observed in figure 12 .

From the background neutral density profiles, we can also derive the sink of all neutrals in the plasma, which corresponds to the plasma ionization source $S$. The formulas to derive $S$ are:

$$
\begin{gathered}
\Gamma_{0}=\frac{1}{4} n_{0}\left\langle v_{0}\right\rangle=n_{0} \sqrt{\frac{k_{B} T_{0}}{2 \pi m_{0}}}, \\
S=\frac{\partial n_{0}}{\partial t}+\nabla \Gamma_{0} \approx \frac{\partial \Gamma_{0}}{\partial r}
\end{gathered}
$$

where $\Gamma_{0}$ is the total background neutral flux in direction from the edge towards the core simplified as a one direction flux through the planar surface, which is acceptable close to the separatrix, $\left\langle v_{0}\right\rangle$ is the neutrals average kinetic velocity, $k_{\mathrm{B}}$ is the Boltzmann constant, $T_{0}$ is the local background neutral temperature, which we assume is the same as ion temperature $T_{0}=T_{\mathrm{i}}$. The time derivative of reconstructed background neutral density $n_{0_{\mathrm{NPA}}}$ is negligible with respect to $\partial \Gamma_{0} / \partial r$.

The evolution of plasma ionization source $S$ over the ELM crash is shown in figure 13 and profiles of $T_{\mathrm{i}}, n_{\mathrm{e}}, S$ and $n_{0}$ with respect to the major radius $R$ in several different times are shown in figure 14.

As the pedestal builds up, before time $t_{1}$ in figure 13 and the blue and green cases in figure 14, the background neutral density $n_{0_{\mathrm{NPA}}}$ is steeper and higher in the SOL and consequently the plasma ionization source $S$ increases in the SOL and the pedestal edge. With the ELM crash, $t_{1} \rightarrow t_{2}$ in figure 13 and the red case in figure 14, the pedestal drops, the ion temperature $T_{\mathrm{i}}$ increases around the separatrix and in the SOL, the separatrix moves inwards and neutrals penetrate deeper. Consequently, plasma ionization source $S$ increases at a constant major radius $R$, but drops with respect to 


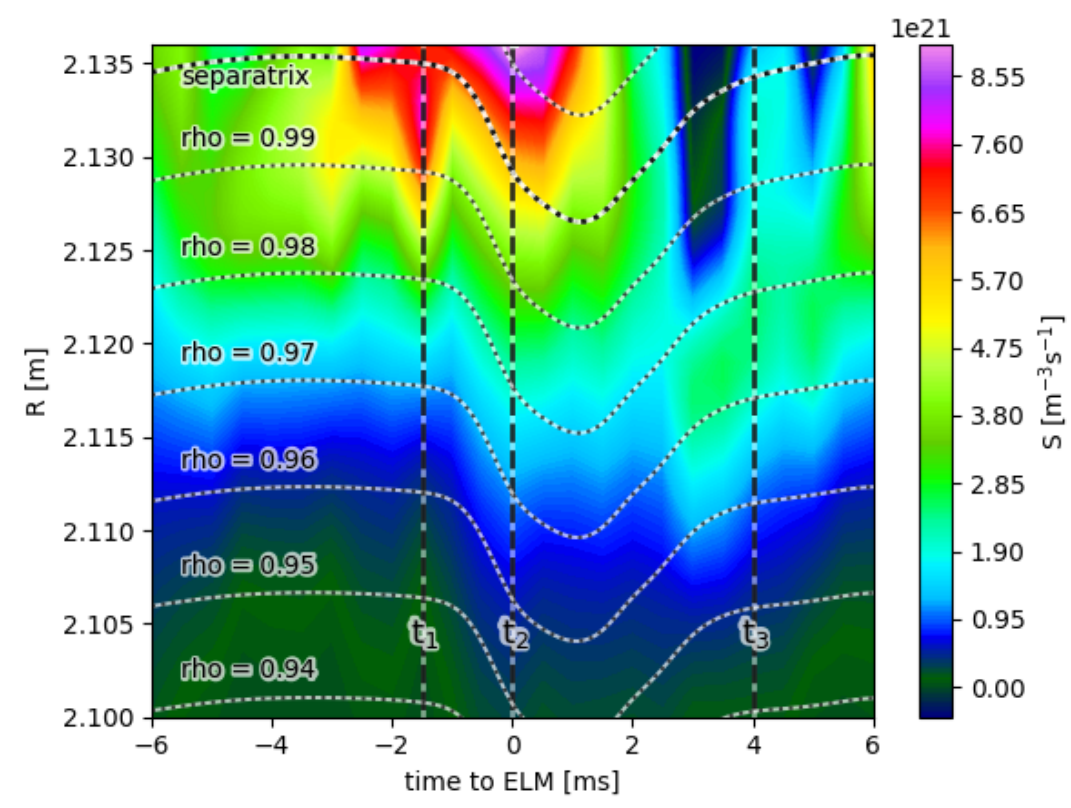

Figure 13. The plasma ionization source $S$ as a function of the time and the major radius $R$. Different $\rho$ positions are illustrated by the gray dashed lines.

the $\rho$ as a result of the separatrix movement. After the ELM, $t_{2} \rightarrow t_{3}$ in figure 13 and the black case in figure 14, the separatrix moves back to the original position and also the plasma density $n_{\mathrm{e}}$ pedestal fully recovers. The recovery of the ion temperature $T_{\mathrm{i}}$ pedestal lags behind plasma density $n_{\mathrm{e}}$ [28]. The position of the reconstructed background neutral density $n_{0_{\mathrm{NPA}}}$ pedestal seems to be driven by the ion temperature $T_{\mathrm{i}}$ pedestal position, because both of them are shifted deeper into the plasma in respect to the plasma density $n_{\mathrm{e}}$. At this point, around $t_{3}$, the SOL background neutral density $n_{0_{\mathrm{NPA}}}$ reaches its minimum. This leads to lower values of the plasma ionization source $S$ after the ELM crash in the separatrix vicinity and higher values deeper in the plasma in comparison with the pre-ELM case.

As discussed in section 3 the assumption of equal neutral and ion temperature $T_{0}=T_{\mathrm{i}}$ is acceptable only inside the separatrix. There are systematic uncertainties of reconstructed background neutral density $n_{0_{\mathrm{NPA}}}$, which lead to the systematic underestimation of $n_{0_{\mathrm{NPA}}}$ in the SOL. They are partially balanced by the overestimation of neutral temperature $T_{0}\left(T_{0}<T_{\mathrm{i}}\right)$ in case of the total neutral flux $\Gamma_{0}$, but we have to keep in mind the fact that reliability of plasma ionization source $S$ in SOL is weaker.

\section{Summary and conclusion}

We have demonstrated the possibility of reconstructing background neutral density profiles based on passive thermal neutral fluxes measured by NPAs. The background neutral density profile can be obtained in absolute units when absolutely calibrated NPA data are provided. In case of uncalibrated NPA data, the shape of the reconstructed 


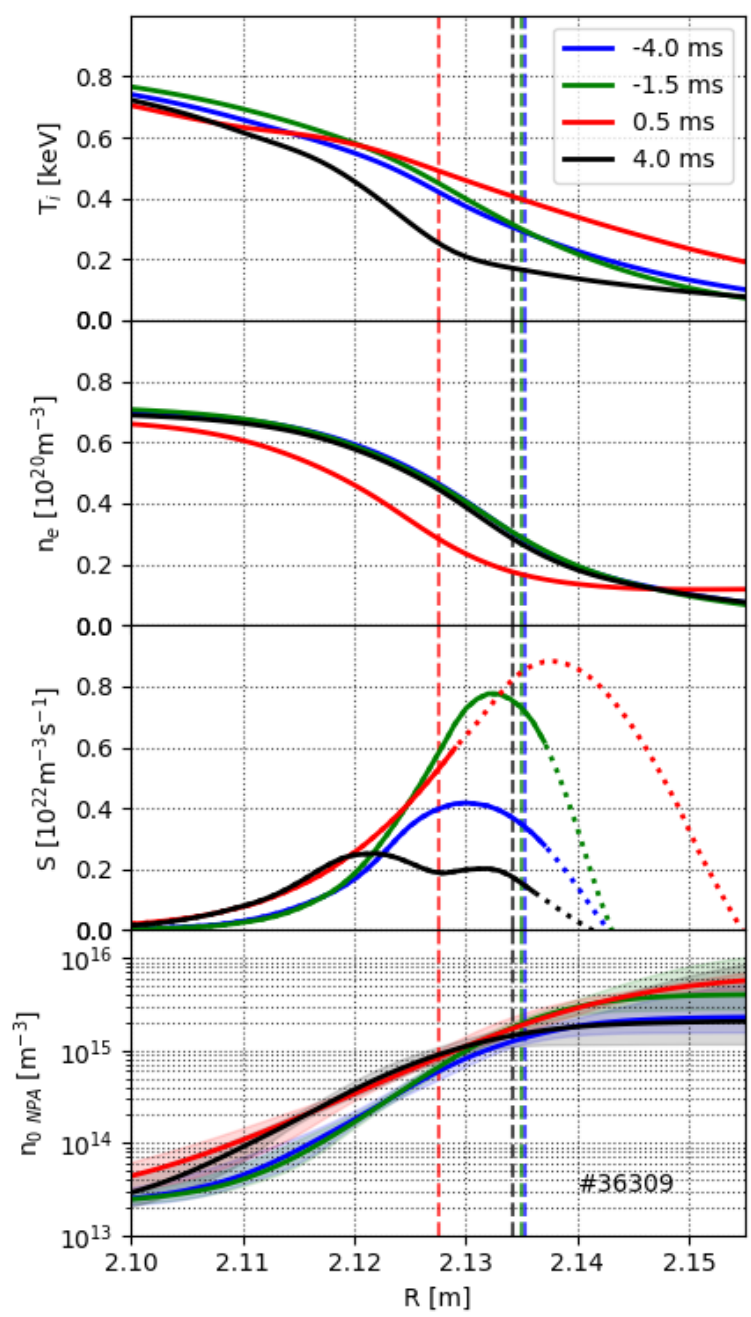

Figure 14. Ion temperature $T_{\mathrm{i}}$, plasma density $n_{\mathrm{e}}$, plasma ionization source $S$ and reconstructed background neutral density $n_{0_{\mathrm{NPA}}}$ profiles at the pedestal are shown by solid lines and separatrix position from equilibrium reconstruction is represented by dashed lines. The different colours stand for different times of the ELM cycle. The reliability of the determined plasma ionization source $S$ is dropping in the SOL due to the assumption that neutrals have the same temperature as plasma ions, which is inaccurate in the SOL. Therefore they are shown only by dotted lines.

background neutral density profile is still reliable. The method was benchmarked against the measured NPA data employing synthetic diagnostic FIDASIM, which uses the reconstructed background neutral density profile as an input. The assumptions that the neutral and ion temperatures are equal and that the effective charge is constant do only impose uncertainties in the range of $\sim 5 \%$ inside the confined region.

There is a wide range of possible applications of the reconstructed neutral density profiles. The high-energy NPA signal could, for instance, be recovered by FIDASIM using the reconstructed neutral density and a TRANSP-predicted fast ion distribution. This will allow detailed fast-ion transport studies based on passive and absolutely calibrated NPA measurements. Moreover, we have presented the profile evolution during 
an ELM cycle. It was shown, that despite a previously measured increase of the midplane wall gas pressure $p_{0}$ [27], the background neutral density can actually drop with the ELM crash. This is caused by the separatrix movement and an increase of the neutral ionization rate in the SOL due to the released plasma, which surpasses the increase in the neutral source. A drop of the neutral density during the ELM recovery phase is expected and also observed in lower energy channels of the NPA.

With knowledge of the background neutral density profiles we can, for the first time, provide a local measurement of the plasma ionization source $S$ in the pedestal region, which was typically a missing quantity in diffusion studies. When the pedestal is fully built up, high gradients in plasma quantities $T_{\mathrm{i}}$ and $n_{0_{\mathrm{NPA}}}$ lead to a significant plasma ionization source $S$ around the separatrix. With the ELM crash, the separatrix moves inwards and the profiles of $T_{\mathrm{i}}$ and $n_{0_{\mathrm{NPA}}}$ flatten, while the background neutral density $n_{0_{\mathrm{NPA}}}$ increases at a given major radius position $R$. The plasma ionization source $S$ as a function of $\rho$ therefore drops, despite it is increasing at constant $R$. During the ELM recovery phase, the plasma ionization source $S$ features a minimum at $R \sim 2.12 \mathrm{~m}$, corresponding to $\rho \sim 0.97$. This is explained by a reduction of the background neutral density $n_{0_{\mathrm{NPA}}}$ in the SOL, persistently high values inside the confined region and a shifted ion temperature $T_{\mathrm{i}}$ pedestal towards the plasma. Based on this analysis, it seems that the plasma ionization source $S$ and also the background neutral density profile shape and magnitude is strongly driven by the ion temperature $T_{\mathrm{i}}$. This underlines that high quality ion temperature $T_{\mathrm{i}}$ data is mandatory for this analysis.

It has to be noted that our conclusions about the background neutral density $n_{0_{\mathrm{NPA}}}$ and plasma ionization source $S$ evolution over the ELM cycle are only valid for this individual discharge. The background neutral density following the ELM crash could also increase if the magnitude of the wall gas density $n_{\mathrm{D}_{2}}$ overcame the impact of higher ionization rate. Therefore, each discharge has to be studied individually.

There are several ways to improve the quality of the reconstructed background neutral density $n_{0_{\mathrm{NPA}}}$ by the described method. First, the range of measured energies of the thermal fluxes can be optimized for expected discharge parameters. Second, information about the SOL neutral temperature $T_{0}$, together with low energy NPA measurements would provide detailed information about the SOL background neural density and plasma ionization source. Third, increasing the number of measured NPA points would open the possibility to improve the complexity of the function used to describe the background neutral density profile.

\section{Acknowledgments}

This work has been carried out within the framework of the project COMPASS-U: Tokamak for cutting-edge fusion research (No. CZ.02.1.01/0.0/0.0/16_019/0000768) and co-funded from European structural and investment funds and of the EUROfusion Consortium and has received funding from the Euratom research and training programme 2014-2018 and 2019-2020 under grant agreement No 633053. The views and 
opinions expressed herein do not necessarily reflect those of the European Commission.

\section{References}

[1] Geiger B, Karpushov A, Duval B, Marini C, Sauter O, Andrebe Y, Testa D, Marascheck M, Salewski M, Schneider P et al. 2017 Plasma Physics and Controlled Fusion 59115002

[2] Gordeev I, Zinovev A and Petrov M 1977 JETP Letters 25 204-207

[3] Haas G and Bosch H 1998 Vacuum 51 39-46

[4] Stotler D, Scotti F, Bell R, LeBlanc B and Raman R 2015 Journal of Nuclear Materials 463 897-901

[5] Boivin R, Hughes J, LaBombard B, Mossessian D and Terry J 2001 Review of Scientific Instruments 72 961-964

[6] Afrosimov V, Gladkovskii I, Gordeev Y S, Kalinkevich I and Fedorenko N 1960 Zhur. Tekh. Fiz. 30

[7] McDermott R, Lebschy A, Geiger B, Bruhn C, Cavedon M, Dunne M, Dux R, Fischer R, Kappatou A, Pütterich T et al. 2017 Review of Scientific Instruments 88073508

[8] Cavedon M, Pütterich T, Viezzer E, Dux R, Geiger B, McDermott R, Meyer H, Stroth U and Team A U 2017 Review of Scientific Instruments 88043103

[9] Chrystal C, Burrell K, Grierson B A, Haskey S R, Groebner R, Kaplan D H and Briesemeister A 2016 Review of Scientific Instruments 87 11E512

[10] Medley S, Donné A, Kaita R, Kislyakov A, Petrov M and Roquemore A 2008 Review of Scientific Instruments $\mathbf{7 9} 011101$

[11] Geiger B, Stagner L, Heidbrink W W, Dux R, Fischer R, Fujiwara Y, Garcia A, Jacobsen A S, Jansen vanVuuren A, Karpushov A N et al. 2020 Plasma Physics and Controlled Fusion

[12] Medley S, Bell R, Petrov M, Roquemore A and Suvorkin E 2003 Review of scientific instruments 74 1896-1899

[13] Karpushov A N, Duval B P, Schlatter C, Afanasyev V I and Chernyshev F V 2006 Review of Scientific Instruments $\mathbf{7 7} 033504$

[14] Varje J, Sirén P, Weisen H, Kurki-Suonio T, Äkäslompolo S et al. 2017 Journal of Instrumentation $12 \mathrm{C} 11025$

[15] Beiersdorfer P, Roquemore A and Kaita R 1987 Review of scientific instruments 58 2092-2098

[16] Mitosinkova K, Melnik A, Tomes M, Stockel J, Janky F, Komm M, Imrisek M, Hacek P, Varju J and Weinzettl V 2016 First results from the neutral particle analyzer on the compass tokamak 1st EPS conference on Plasma Diagnostics vol 240 (SISSA Medialab) p 074

[17] Salvatier J, Wiecki T V and Fonnesbeck C 2016 PeerJ Computer Science 2 e55

[18] Boivin R, Goetz J, Hubbard A, Hughes J, Hutchinson I, Irby J, LaBombard B, Marmar E, Mossessian D, Pitcher C et al. 2000 Physics of Plasmas 7 1919-1926

[19] Salewski M, Geiger B, Jacobsen A, Abramovic I, Korsholm S, Leipold F, Madsen B, Madsen J, McDermott R, Moseev D et al. 2018 Nuclear Fusion 58036017

[20] LaBombard B 2001 KN1D: A 1-D space kinetic, 2-D velocity, transport algorithm for atomic molecular hydrogen in an ionizing plasma Research Report PSFC-RR-01-3 Massachusetts Institute of Technology, Plasma Science and Fusion Center 175 Albany St., Cambridge, MA 02139 USA

[21] Geiger B, Weiland M, Mlynek A, Reich M, Bock A, Dunne M, Dux R, Fable E, Fischer R, GarciaMunoz M et al. 2014 Plasma Physics and Controlled Fusion 57014018

[22] Zohm H 1996 Plasma Physics and Controlled Fusion 38105

[23] Fischer R, Fuchs C, Kurzan B, Suttrop W, Wolfrum E and Team A U 2010 Fusion science and technology $\mathbf{5 8} 675-684$

[24] Denk S S, Fischer R, Smith H, Helander P, Maj O, Poli E, Stober J, Stroth U, Suttrop W, Westerhof E et al. 2018 Plasma Physics and Controlled Fusion 60105010

[25] Kurzan B and Murmann H 2011 Review of scientific instruments 82103501 
[26] Haas G and Bosch H 1998 Vacuum 51 39-46

[27] Laggner F, Keerl S, Gnilsen J, Wolfrum E, Bernert M, Carralero D, Guimarais L, Nikolaeva V, Potzel S, Cavedon M et al. 2018 Plasma Physics and Controlled Fusion 60025002

[28] Cavedon M, Pütterich T, Viezzer E, Laggner F, Burckhart A, Dunne M, Fischer R, Lebschy A, Mink F, Stroth U et al. 2017 Plasma Physics and Controlled Fusion 59105007 